\title{
Electronic Shell Structure of Nanoscale Superconductors
}

\author{
K. Tanaka and F. Marsiglio \\ Department of Physics, University of Alberta, Edmonton, Alberta, Canada T6G 2J1
}

(November 6, 2018)

\begin{abstract}
Motivated by recent experiments on $\mathrm{Al}$ nanoparticles, we have studied the effects of fixed electron number and small size in nanoscale superconductors, by applying the canonical BCS theory for the attractive Hubbard model in two and three dimensions. A negative "gap" in particles with an odd number of electrons as observed in the experiments is obtained in our canonical scheme. For particles with an even number of electrons, the energy gap exhibits shell structure as a function of electron density or system size in the weak-coupling regime: the gap is particularly large for "magic numbers" of electrons for a given system size or of atoms for a fixed electron density. The grand canonical BCS method essentially misses this feature. Possible experimental methods for observing such shell effects are discussed.
\end{abstract}

PACS number(s): 74.20.Fg, 71.24.+q, 71.10.Fd, 71.10.Li

As the technology for fabricating ultrasmall metallic grains steadily improves, the typical sample dimensions are approaching molecular dimensions [1],2]. The present accessibility to samples ranging from nanoscale to bulk has renewed interest in various features of the solid state that may or may not survive the excursion to ultrasmall dimensions. In superconducting Al samples, for example, the ability to distinguish even and odd numbers of electrons through tunneling experiments [3] 5] has called into question the use of the grand canonical ensemble to describe the electron pairing in these ultrasmall samples within a model with equal level spacings [6], and within the attractive Hubbard model [7]. In these works the BCS theory of pairing was formulated within the canonical ensemble, following the early treatment of nuclei [8], and some rigorous "quality control" was provided by exact studies [9,7,10,11].

Within the attractive Hubbard model we have found two prominent features that emerged from the canonical BCS treatment, both of which were verified by the exact solutions. The first is the existence of "negative gaps" for odd electron number grains. By this we simply mean that a tunneling bias less than the charging energy would be required to tunnel an electron onto a grain with an odd number of electrons. The second is the existence of what were termed "super-even" electron numbers, where the tunneling bias required to tunnel an electron onto a grain with certain even numbers of electrons would be unusually high. In this letter we investigate these features for various bandstructures in two and three dimensions, as might apply to $\mathrm{Al}$, and briefly discuss some possible experiments to observe in particular the "super-even" effect.

We have adopted the attractive Hubbard model, whose specifics are well known. The additional feature we include here is the possibility of using both periodic boundary conditions (PBC) as well as open boundary conditions (OBC), which are more appropriate for small systems. Either of these is accomplished through a unitary transformation to a basis that diagonalizes the kinetic energy term. The BCS variational calculation is then performed with a wave function containing pairs of timereversed states $(n, \uparrow)$ and $(n, \downarrow)$ [12]. The even and odd wave functions with $\nu$-pairs are given by 8

$$
\begin{aligned}
\left|\Psi_{2 \nu}\right\rangle & =c \frac{1}{2 \pi i} \oint d \xi \xi^{-\nu-1} \prod_{n}\left(1+\xi g_{n} a_{n \uparrow}^{\dagger} a_{n \downarrow}^{\dagger}\right)|0\rangle \\
\left|\Psi_{2 \nu+1}\right\rangle & =c \frac{1}{2 \pi i} \oint d \xi \xi^{-\nu-1} a_{m \sigma}^{\dagger} \prod_{n \neq m}\left(1+\xi g_{n} a_{n \uparrow}^{\dagger} a_{n \downarrow}^{\dagger}\right)|0\rangle,
\end{aligned}
$$

with $N_{e}=2 \nu$ and $N_{e}=2 \nu+1$, respectively. The contour integral is on any counterclockwise path that encloses the origin. For odd $N_{e}$, the blocked state $m$ is chosen so that it gives the lowest energy for a given coupling strength. Details with $\mathrm{PBC}$ were given previously [7], and with $\mathrm{OBC}$ they will be given elsewhere. We calculate the ground state energy for three systems with electron number $N_{e}, N_{e}+1$, and $N_{e}+2$, and evaluate the energy gap by the formula $\Delta_{N_{e}}=\left(E_{N_{e}-1}-2 E_{N_{e}}+E_{N_{e}+1}\right) / 2$.

In the grand canonical ensemble, the number of electrons is fixed only on average. Thus we must solve the gap equation,

$$
\Delta_{n}=\sum_{m}\left(\operatorname{Re} V_{n n, m m}\right) \frac{\Delta_{m}}{2 E_{m}}
$$

along with the number equation,

$$
n_{e} \equiv \frac{\left\langle N_{e}\right\rangle}{N}=1-\frac{1}{N} \sum_{n} \frac{1}{E_{n}}\left(\tilde{\epsilon}_{n}-\mu\right)
$$

for gap parameters $\left\{\Delta_{n}\right\}$ and chemical potential $\mu$. Here, $V_{n n, m m}$ is the transformed interaction, $E_{n} \equiv$ $\sqrt{\left(\tilde{\epsilon}_{n}-\mu\right)^{2}+\Delta_{n}^{2}}$ is the quasiparticle energy and $\tilde{\epsilon}_{n}=$ $\epsilon_{n}+\sum_{m} V_{n m, n m} \frac{g_{m}^{2}}{1+g_{m}^{2}}$ is the single-particle energy modified with the Hartree term. The gap is given by $\Delta_{0}=$ $\min \left(E_{n}\right)$ for a finite size system, that is, with quantized energy levels $\left\{\epsilon_{n}\right\}$.

In the weak to intermediate coupling regime, the energy gap as a function of electron number (or electron 
density $n_{e}$ ) roughly reflects the single-particle density of states (DOS). Thus in a simple cubic (SC) lattice in three dimensions (3D), the gap in the bulk limit is a smooth function of $n_{e}$ that increases from zero at zero density 113] to a maximum value at half filling. In Fig. 1(a) we show the single-electron DOS for a SC lattice of $N=16 \times 16 \times 16=4096$ sites with PBC (solid curve) . The result has been smoothed by convolution with a normalized Gaussian, and it is very similar to the bulk density of states shown by the dashed curve. Although $N=4096$ is fairly large, the energy gap behaves quite differently from what we expect for a bulk system as a function of electron density for weak coupling. Results for the grand canonical BCS gap, $\Delta_{0} / t$, are illustrated in Fig. 11(b) for $|U| / t=2$ and (c) for $|U| / t=1$. For $|U| / t=2$ the overall scale of the gap $\Delta_{0}$ as a function of $n_{e}$ resembles $g(\epsilon)$ shown in Fig. 11(a). However, it has many fine structures; discontinuities at small density and cusps at larger density. This non-smooth behaviour is a result of the discrete density of states, i.e., quantized energy levels $\left\{\epsilon_{n}\right\}$ and their degeneracy in a finite size system. Such quantum structures of $\Delta_{0}$ turn out to be prominent for weaker coupling strengths, as seen for $|U| / t=1$ in Fig. 1 1 (c): in this case there are discontinuities in the gap for the entire range of density. The
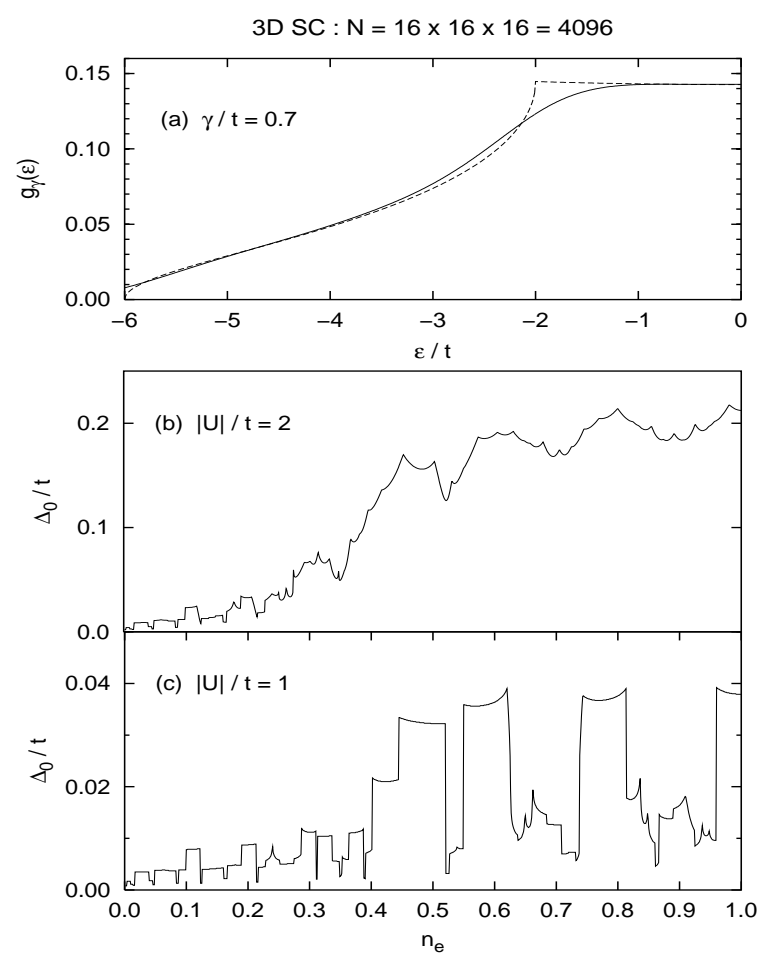

FIG. 1. (a) Density of states smoothed by Gaussian convolution with width $\gamma=0.7 t$ for $3 \mathrm{D} N=16^{3}=4096$ sites (solid curve). The dashed curve is the bulk density of states. (b) Energy gap obtained by the grand canonical BCS, $\Delta_{0} / \mathrm{t}$, as a function of electron density $n_{e}$ for $N=16^{3}=4096$ sites and $|U| / t=2$. (c) Same as (b) but for $|U| / t=1$. discontinuities or cusps in the gap arise from finite level spacings, while their positions as a function of $n_{e}$ and the magnitude of the gap are determined by the degeneracy of levels, as will be explained in detail shortly.

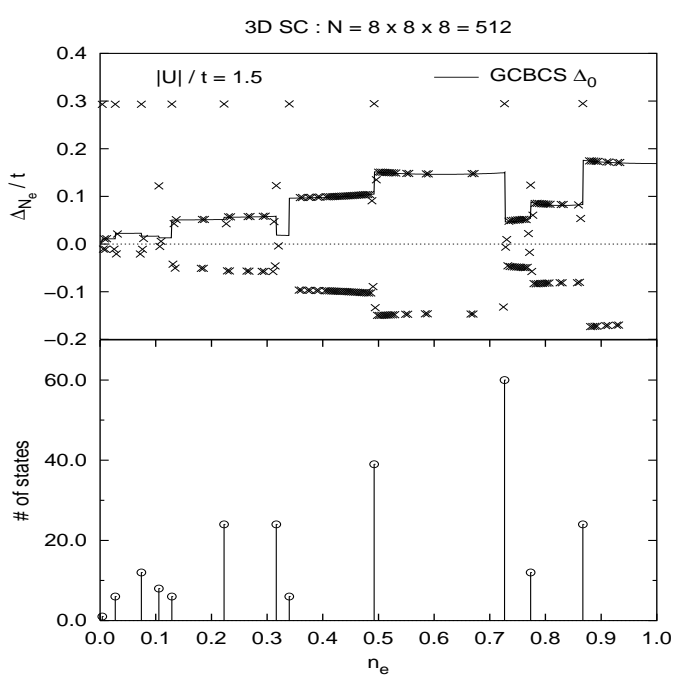

FIG. 2. Energy gap $\Delta_{N_{e}} / \mathrm{t}$ from the canonical BCS (crosses) and $\Delta_{0} / \mathrm{t}$ from the grand canonical BCS (solid curve) as a function of electron density $n_{e}$ for $3 \mathrm{D}$ $N=8^{3}=512$ sites and $|U| / t=1.5$ (upper frame): the canonical results are shown for only some representative densities. The number of single-particle states (spin degeneracy not included) as a function of $n_{e}$ (lower frame): each of the discrete levels is plotted at the density that corresponds to the closed-shell configuration up to that level in the zero-coupling limit. The canonical gap $\Delta_{N_{e}} / \mathrm{t}$ for weak coupling has jumps at these densities as seen in the upper figure, while the height of these jumps is determined by the energy spacing to the next level.

In Fig. 2 (upper frame) the gap $\Delta_{N_{e}}$ obtained by the canonical BCS is shown with crosses, along with the grand canonical gap $\Delta_{0}$ (solid curve), as a function of electron density $n_{e}$, for a nanoscale system in weak coupling. The most obvious new feature is the "negative gap", for systems with an odd number of electrons. As was already mentioned, this result has already been observed in small $\mathrm{Al}$ grains [5]. For the even numbered grains note that most of the results shown follow the discontinuous, step-function-like behaviour of $\Delta_{0}$. However, anomalously high values occur at densities where the grand canonical result has discontinuities. These anomalies follow from the analogue of shell effects for a finite lattice of electrons. In the lower part of Fig. 2 we plot the number of single-particle states as a function of $n_{e}$ for this system. Each of the discrete levels is plotted at the density that corresponds to electron number for filling all the levels up to that particular level ("closed- 
shell" configuration [14) and the height is the degeneracy of the level without the spin factor of two. It is clear that the densities where the canonical gap has a jump are the ones that correspond to the closed-shell configurations.

In a closed-shell configuration, the occupation of levels is mainly driven by the kinetic energy. The cost required to occupy higher energy states exceeds the gain due to the increased interaction. Moreover, a careful examination of the energy gain due to pairing reveals two distinct sources, a Hartree-like term, and the explicit BCS pairing term. The latter is small compared to the former, so the loss in energy reduction due to less mixing of states is indeed quite small. The Hartree-like term continues to play a role, however, which is why the value of the gap in the closed shell configurations is approximately equal to half the level spacing (i.e. one would have expected an additional pairing energy). The same physics occurs within the grand canonical ensemble [7], though the discontinuity is the best these equations can do to account for the closed shell configurations.
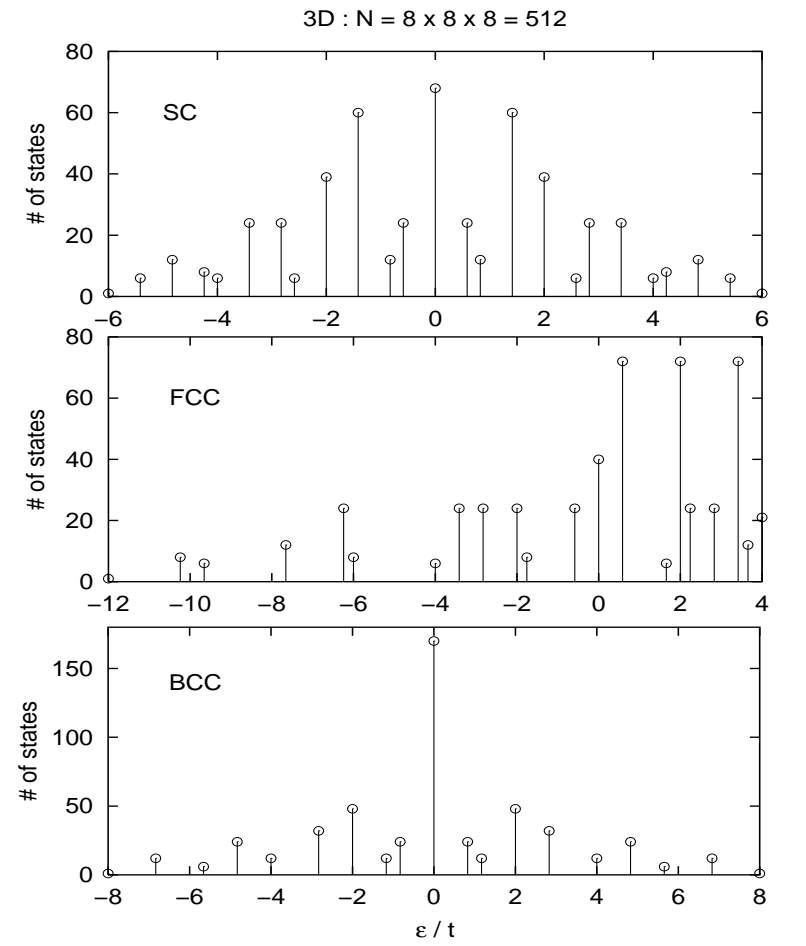

FIG. 3. The number of states (spin degeneracy not included) as a function of single-particle energy $\epsilon$ for $3 \mathrm{D}$ $N=8^{3}=512$ sites for different lattice structures; SC (upper frame), FCC (middle frame), and BCC (bottom frame). The $\mathrm{SC}$ and BCC level structures have particle-hole symmetry, while FCC does not.

Different level structures result in different shell structures in the gap. In Fig. 3 we show the number of states for $N=8^{3}=512$ sites with PBC for SC, FCC (facecentred cubic) and BCC (body-centred cubic) lattices, as a function of single-particle energy for the entire band. The one for SC (top frame) for negative energy is the same as shown in Fig. 2, except now it is plotted vs. energy so that the level spacings are clearly visible; these in turn determine the height of the jumps in the canonical gap. In fact for such a small system there are only two distinct level spacings in SC. This is why in the gap shown in Fig. 2, there are only two anomalously high values for the gap. In FCC (middle frame) there is no particle-hole symmetry and the degeneracy is more concentrated near the top of the band. Compared with SC, the jumps at closed shell configurations will be more enhanced by the larger level spacings and more frequent for smaller density; in addition the gap for open shells will be smaller (on average) because of less degeneracies. The BCC (bottom frame) has particle-hole symmetry as in $\mathrm{SC}$, but the degeneracy is concentrated around zero energy. As in FCC there will be jumps more frequently at smaller densities, but near half-filling the gap will be continuous with the Fermi level at zero energy.

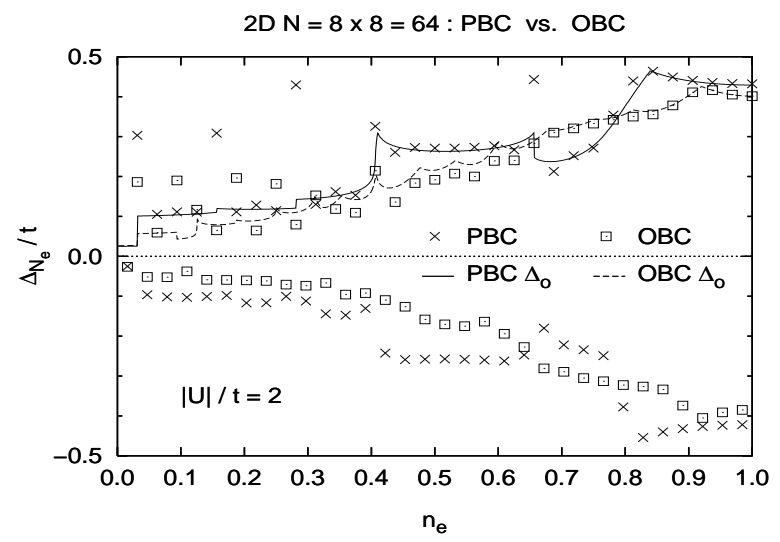

FIG. 4. The canonical energy gap $\Delta_{N_{e}} / \mathrm{t}$ with PBC (crosses) and $\mathrm{OBC}$ (squares) and the grand canonical $\Delta_{0} / \mathrm{t}$ with PBC (solid curve) and OBC (dashed curve) as a function of electron density $n_{e}$ for $2 \mathrm{D} N=8^{2}=64$ sites and $|U| / t=2$. With $\mathrm{OBC}$, due to lower symmetry and hence less degeneracy of single-particle levels, the canonical gap tends to have more jumps, but with less height.

The level structure also depends on the boundary condition. In Fig. A we illustrate this for a small 2D system, where we compare $\mathrm{PBC}$ and $\mathrm{OBC}$. In Fig. 1 the canonical gap $\Delta_{N_{e}}$ is plotted (for all densities) with crosses (PBC) and squares $(\mathrm{OBC})$, and the grand canonical gap $\Delta_{0}$ is shown with solid (PBC) and dashed (OBC) curves, for $|U| / t=2$. The SC lattice in $2 \mathrm{D}$ with $\mathrm{PBC}$ has a large degeneracy (a singularity in the bulk DOS) at zero singleparticle energy. With OBC a relatively high degeneracy remains at zero energy, but for nonzero energy there are more levels with less degeneracies, because translational 
symmetry is absent. For $N=64$ most of the levels are doubly degenerate, while some have no degeneracy. This is why in Fig. 14 the canonical gap with OBC has jumps more frequently (often periodic between $N_{e}$ multiples and non-multiples of four) with less height than with PBC at smaller density. We note again that the canonical gap for even $N_{e}$ for open shells and that for odd $N_{e}$ look symmetric about the x-axis (for both $\mathrm{PBC}$ and $\mathrm{OBC}$ ).

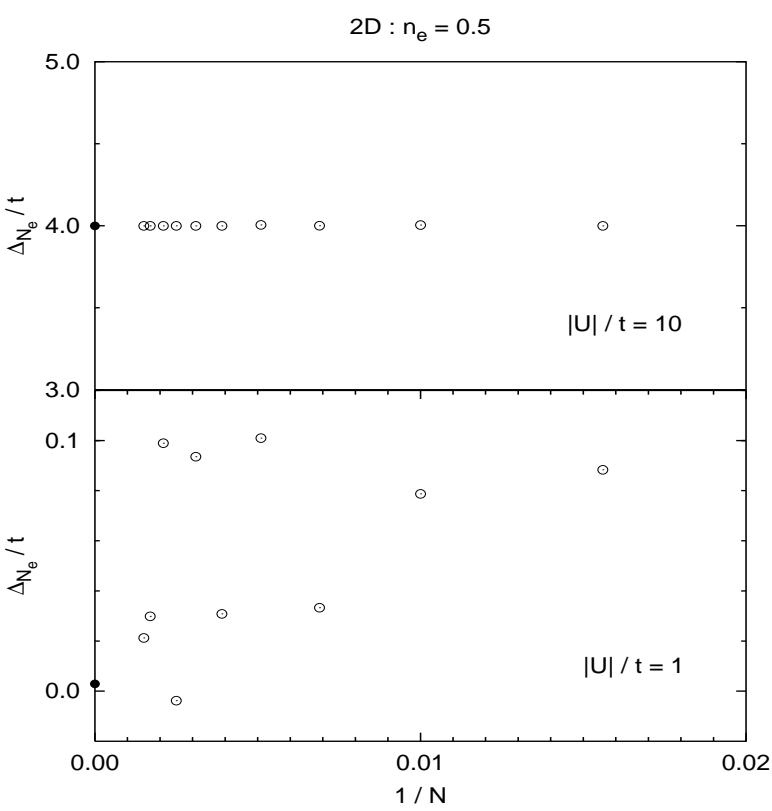

FIG. 5. The canonical gap $\Delta_{N_{e}} / t$ (circles) for $2 \mathrm{D} N=N_{s}^{2}$ sites for quarter filling $n_{e}=0.5$ as a function of $1 / N$; for $N_{s}=8,10,12,14,16,18,20,22,24,26$ and for $|U| / t=10$ (upper frame) and $|U| / t=1$ (lower frame). The grand canonical gap $\Delta_{0} / t$ for $N_{s}=1000$ is shown on the ordinate with a filled circle.

We can also see the shell effects for a fixed electron density by varying the lattice size. In Fig. 5 the canonical gap $\Delta_{N_{e}}$ is shown as a function of $1 / N\left(N \equiv N_{s}^{2}\right)$ for a 2D SC lattice with $\mathrm{PBC}$ for quarter filling $n_{e}=0.5$ (circles), for strong coupling (upper frame) and weak coupling (bottom frame). The grand canonical gap in the bulk limit is indicated with a solid circle on the ordinate. In strong coupling the gap hardly depends on the number of sites. In weak coupling the gap exhibits strong size dependence as can be seen for $|U| / t=1$. For $N_{s}=10,14,18,22$ and 26, quarter filling corresponds to a closed-shell configuration and this can be seen clearly for $N_{s}=14,18$ and 22 as the big jumps, which reflect the level spacing in each case. In contrast the Fermi level is open for $N_{s}=8,12,16,20,24$. Interestingly the gap for open shells also changes as a function of size in a non-smooth way [15]. By far, however, the transition to the bulk regime is dominated by the oscillations of the magnitude of the gap between open and closed shell configurations.

In summary, we have examined the tunneling gap for three dimensional ultrasmall superconducting grains, as a function of electron density, coupling strength, and system size. In weak coupling, shell effects are particularly prominent, and should be observable in very clean grains at low temperature. An ideal experimental arrangement would allow one to vary the electron density over a wide range. In this way one could observe the large modulation of the gap and identify "magic numbers" of electrons corresponding to the electron densities with anomalously large gap. However, in practice we anticipate that through the use of a gate electrode, one can vary the electron density only by a small amount (though large enough to see even/odd effects [5]). Hence one will have to rely on ion-implanting a distribution of grain sizes, and thus make use of Fig. 5 to correlate gaps of different magnitude with different grain sizes. A systematic search should yield grain sizes whose electron number lies near a "magic number" so that tunneling a handful of electrons (one by one) onto the sample controlled by a gate electrode will allow one to observe large changes in the gap, as illustrated in Fig. 2.

We thank Allen Goldman, Boldizsár Jankó, and $\mathrm{Al}$ Meldrum for enlightening discussions on possible experimental methods. Calculations were performed on the 44-node SGI parallel processor at the University of Alberta. This research was supported by the Avadh Bhatia Fellowship and by the Natural Sciences and Engineering Research Council of Canada and the Canadian Institute for Advanced Research.

[1] D. Davidović and M. Tinkham, cond-mat/9811259.

[2] D. Davidović and M. Tinkham, cond-mat/9905043.

[3] M. T. Tuominen, J. M. Hergenrother, T.S. Tighe and M. Tinkham, Phys. Rev. Lett. 69, 1997 (1992).

[4] P. Lafarge, P. Joyez, D. Esteve, C. Urbina and M. H. Devoret, Phys. Rev. Lett. 70, 994 (1993).

[5] D. C. Ralph, C. T. Black and M. Tinkham, Phys. Rev. Lett. 74, 3241 (1995); Phys. Rev. Lett. 76, 688 (1996); Phys. Rev. Lett. 78, 4087 (1997).

[6] F. Braun and J. von Delft, Phys. Rev. Lett. 81, 4712 (1998).

[7] K. Tanaka and F. Marsiglio, Phys. Rev. B 60, xxxx (1999).

[8] K. Dietrich, H. J. Mang and J. H. Pradal, Phys. Rev. 135, B22 (1964).

[9] A. Mastellone, G. Falci, and R. Fazio, Phys. Rev. Lett. 80, 4542 (1998).

[10] J. Dukelsky and G. Sierra, cond-mat/9903332, condmat/9906166. 
[11] R.W. Richardson and N. Sherman, Nucl. Phys. 52, 221 (1964); R.W. Richardson, Phys. Rev. 141, 949 (1966).

[12] P. W. Anderson, J. Phys. Chem. Solids 11, 26 (1959).

[13] We leave aside here the interesting issue of a minimum density required in weak coupling and three dimensions for a bound state, and the interesting possibility of a bound state existing in an ultrasmall system where none exists in the bulk.

[14] D. Bormann, T. Schneider, and M. Frick, Z. Phys. B 87, 1 (1992).

[15] In 1D we have found the canonical gap for open shells ( $N$ multiples of four) and that for closed shells ( $N$ nonmultiples of four) are both smooth functions of $1 / N$ separately (unpublished). The "crossover" behaviour of the canonical gap has been discussed as a function of level spacing for a model with uniformly spaced levels [16, 9, 10]. The crossover in the canonical BCS results was found to be rather sharp for this model [6].

[16] K. A. Matveev and A. I. Larkin, Phys. Rev. Lett. 78, 3749 (1997). 